\title{
ON A PROBLEM OF M. P. SCHÜTZENBERGER
}

\author{
by D. B. McALISTER $\dagger$
}

(Received 17th May 1978)

A class of finite semigroups is called a genus if it is closed under homomorphic images, subsemigroups and finite direct products. During a talk at the Symposium on Semigroups held at the University of St Andrews, in 1976, M. P. Schützenberger posed the problem of characterising the smallest genus $\mathscr{G}$ which contains finite groups and finite semigroups, all of whose subgroups are trivial.

If $D \in \mathscr{G}$ then, as pointed out by Schützenberger, the subsemigroup $I G(S)$, generated by the idempotents of $S$, has only trivial subgroups. In the first section of this note we deduce a property of the members of $\mathscr{G}$ which may be used to show that the converse is false. This property also shows that, if a finite regular semigroup $S$ belongs to $\mathscr{G}$, then $\mathscr{H}$ is a congruence on $S$. In the second section we show that, on the other hand if $S$ is orthodox and $\mathscr{H}$ is a congruence, then $S \in \mathscr{G}$. A corollary is that a finite semigroup which is a union of groups belongs to $\mathscr{G}$ if and only if it is an orthodox band of groups.

\section{A necessary condition}

A class of finite semigroups is called a genus if it is closed under homomorphic images, subsemigroups and finite direct products. For example, the class of all finite groups is a genus, as is the class of all finite bands. Another example of a genus is given by the class $\mathscr{A}$ of all finite semigroups $A$ in which each subgroup is trivial. (Following Eilenberg (1), we shall say that a semigroup $A$ is aperiodic if each subgroup of $A$ of trivial). It is easy to see that $\mathscr{A}$ is closed under subsemigroups and finite direct prod ucts. That it is also closed under homomorphic images, is a consequence of the following lemma (c.f. (6)) which is also used later in the paper.

Lemma 1.1. Let $T$ be a finite semigroup and let $\theta$ be a homomorphism of $T$ onto a semigroup $S$. Then, for each subgroup $H$ of $S$ there is a subgroup $K$ of $T$ with $K \theta=H$. Thus, for each idempotent $e \in S$ there is an idempotent $f \in T$ with $f \theta=e$.

Corollary 1.2. Let $\mathscr{A}$ be the class of finite aperiodic semigroups. Then $\mathscr{A}$ is a genus.

Proof. Let $T \in \mathscr{A}$ and let $\theta$ be a homorphism of $T$ onto a semigroup $S$; suppose that $H$ is a subgroup of $S$. Then, by Lemma 1.1 , there is a subgroup $K$ of $T$ with $K \theta=H$. Since $T$ is aperiodic $K$ must be trivial. Hence so is $H$ and therefore $S \in \mathscr{A}$.

$\uparrow$ This research was partially supported by a Grant from the National Science Foundation. 
We have already remarked that $\mathscr{A}$ is closed under subsemigroups and finite direct products. Hence, since it is also closed under homomorphic images, $\mathscr{A}$ must be a genus.

The next corollary will also be useful in what follows.

Corollary 1.3. Let $T$ be a finite semigroup and let $\theta$ be a homorphism of $T$ onto a semigroup $S$. Let $I G(T)$ denote the subsemigroup generated by the idempotents of $T$ and, likewise, let $I G(S)$ denote the subsemigroup generated by the idempotents of $S$. Then $I G(T) \theta=I G(S)$.

Proof. Clearly $\theta$ maps $I G(T)$ into $I G(S)$. On the other hand, suppose that $x=$ $e_{1} e_{2} \ldots e_{n} \in I G(S)$ where $e_{1}, \ldots, e_{n}$ are idempotents. Then, by Lemma 1.1, there are idempotents $f_{1}, \ldots, f_{n} \in T$ with $f_{i} \theta=e_{i}, 1 \leqq i \leqq n$. But then $\left(f_{1} f_{2} \ldots f_{n}\right) \theta=e_{1} e_{2} \ldots e_{n}=x$.

If each of $\mathscr{B}, \mathscr{C}$ is a genus of finite semigroups then, $\operatorname{cf}(\mathbf{1})$, page 110 , a finite semigroup $S$ belongs to the smallest genus which contains $\mathscr{B}$ and $\mathscr{C}$ if and only if $S$ is a homomorphic image of a subsemigroup of $B \times C$ for $B \in \mathscr{B}, C \in \mathscr{C}$. In particular, $S$ belongs to the genus $\mathscr{G}$ generated by finite groups and finite aperiodic semigroups if and only if $S$ is a homomorphic image of subsemigroup of $G \times A$ for some finite group $G$ and finite aperiodic semigroup $A$; that is, $S \in \mathscr{G}$ if and only if $S$ divides $G \times A$.

Lemma 1.4. (Schützenberger). Let $S \in \mathscr{G}$, then the subsemigroup $I G(S)$ of $S$, generated by the idempotents of $S$, is aperiodic.

Proof. Since $S \in \mathscr{G}, S$ divides $G \times A$ for some finite group $G$ and finite aperiodic semigroup $A$. Thus there is a subsemigroup $T$ of $G \times A$ and a homomorphism $\theta$ of $T$ onto $S$.

Each idempotent of $T$ has the form $(1, u)$, where 1 denotes the identity of $G$ and $u$ is an idempotent of $A$. Hence $I G(T) \subseteq\{(1, x): x \in I G(A)\}$. Since $A$ is aperiodic, so is $I G(A)$; thus so is $\{(1, x): x \in I G(A)\}$. Hence $I G(T)$ is aperiodic and hence, by Lemma 1.1 , so is $I G(S)=I G(T) \theta$.

Lemma 1.5. Let $S \in \mathscr{G}$ and let $H$ be a subgroup of $S$, with identity $e$. Then, for each $h \in H, x \in I G(S)$,

$$
h x h^{-1}=\text { exe. }
$$

Proof. Let $T$ be a subsemigroup of $G \times A$ for some finite group $G$ and finite aperiodic semigroup $A$ and let $\theta$ be a homomorphism of $T$ onto $S$. By Lemma 1.1, there is a subgroup $K$ of $T$, with identity $u$, such that $K \theta=H$. Similarly, by Corollary 1.3 , there exists $y \in I G(T)$ such that $y \theta=x$.

Now, since $A$ is aperiodic, $K=K_{1} \times\left\{u_{1}\right\}$ where $K_{1}$ is a subgroup of $G$ and $u_{1}$ is idempotent. Further, since $G$ is a group, $y=\left(1, y_{1}\right)$ where 1 denotes the identity of $G$ and $y_{1} \in I G(A)$. Let $k=\left(k_{1}, u_{1}\right) \in K$ be such that $k \theta=h$. Then

$$
\begin{aligned}
k y k^{-1} & =\left(k_{1}, u_{1}\right)\left(1, y_{1}\right)\left(k_{1}^{-1}, u_{1}\right) \\
& =\left(1, u_{1} y_{1} u_{1}\right)=u y u
\end{aligned}
$$

so that, on applying $\theta$, one gets $h x h^{-1}=$ exe. 
Let $S$ be a regular semigroup and let $e, f$ be idempotents of $S$. Then we denote by $S(e, f)$ the set

$$
S(e, f)=\left\{u^{2}=u \in S: \quad f u=u=u e \quad \text { and } \quad e u f=e f\right\} .
$$

Let $a, b \in S$ with inverse $a^{\prime}, b^{\prime}$ respectively and set $e=a^{\prime} a, f=b b^{\prime}$. Then Nambooripad (5) shows that $S(e, f) \neq \square$ and that $b^{\prime} u a^{\prime}$ is an inverse of $a b$ for each $u \in S(e, f)$.

We shall use these ideas in the proof of the following proposition.

Proposition 1.6. Let $S \in \mathscr{G}$ be a finite regular semigroup. Then $\mathscr{H}$ is a congruence on $S$.

Proof. Let $a, b \in S$ with $a \mathscr{H} b$; then there exists inverses $a^{\prime}$ of $a$ and $b^{\prime}$ of $b$ such that $a a^{\prime}=b b^{\prime}, a^{\prime} a=b^{\prime} b$. Set $h=a b^{\prime}, h^{-1}=b a^{\prime}$; then $h h^{-1}=a b^{\prime} b a^{\prime}=a a^{\prime} a a^{\prime}=a a^{\prime}=b b^{\prime}=$ $b a^{\prime} a b^{\prime}=h^{-1} h$. Thus $h$ belongs to a subgroup of $S$, with identity $e=a a^{\prime}$, and has $h^{-1}$ as inverse there.

Let $x \in S$ and let $x^{\prime}$ be an inverse for $x$. Pick $u \in S\left(a^{\prime} a, x x^{\prime}\right)$; then $x^{\prime} u a^{\prime}$ is an inverse for ax. Now

$$
\begin{array}{rlrl}
a x . x^{\prime} u a^{\prime} & =a x x^{\prime} u a^{\prime} & & \\
& =a u a^{\prime} & & \text { since } u \in S\left(a^{\prime} a,\right. \\
& =a a^{\prime} a u a^{\prime} a a^{\prime} & & \\
& =a b^{\prime} b u b^{\prime} b a^{\prime} & & \text { where } y=b u b^{\prime} . \\
& =h y h^{-1} &
\end{array}
$$

Now $b u b^{\prime} . b u b^{\prime}=b u a^{\prime} a u b=b u^{2} b^{\prime}=b u b^{\prime}$ since $u \in S\left(a^{\prime} a, x x^{\prime}\right)$. Thus, by Lemma 1.2,

$$
h y h^{-1}=e y e=a a^{\prime} b u b^{\prime} a a^{\prime}=b u b^{\prime}=b x \cdot x^{\prime} u b^{\prime} .
$$

Hence $a x \mathscr{R} b x$ and, clearly $a x \mathscr{L} b x$; thus $a x \mathscr{H} b x$. Similarly $x a \mathscr{H} x b$ so that $\mathscr{H}$ is a congruence.

Corollary 1.7. For any $n \geqq 2$, the symmetric inverse semigroup $\Phi_{n}$ on $n$ letters does not belong to $\mathscr{G}$.

Proof. $\mathscr{I}_{n}$ is fundamental inverse semigroup (4) but $\mathscr{H}$ is not a congruence on $I_{n}$. Hence $I_{n}$ does not belong to $\mathscr{G}$.

The idempotents in an inverse semigroup $S$ commute so they form a subsemigroup of $S$. Hence $I G(S)$ consists entirely of idempotents and so is aperiodic. Since $I_{n}, n \geqq 2$ does not belong to $\mathscr{G}$ it follows the converse of Lemma 1.4 is false.

\section{Orthodox semigroups}

In this section we prove that, if $S$ is a finite orthodox semigroup on which $\mathscr{H}$ is a congruence, then $S \in \mathscr{G}$.

A regular semigroup $S$ is called $E$-unitary if the idempotents form a unitary subset of $S$. Equivalently, $S$ is $E$-unitary if the idempotents form a class of some group congruence (the minimum group congruence) on $S$.

Lemma 2.1. Let $S$ be an orthodox semigroup. Then there is an $E$-unitary semigroup $T$ and an idempotent separating homomorphism of $T$ onto $S$. If $S$ is finite, $T$ can also be chosen to be finite. 
Proof. Hall (2) has shown that $S$ is a subdirest product of $S / \mu$ and $S / \mathscr{Y}$ where $\mu$ is the maximum idempotent separating congruence on $S$ and $\mathscr{Y}$ is the minimum inverse semigroup congruence on $S$. By (3), there is an $E$-unitary inverse semigroup $V$ and an idempotent separating homomorphism $\phi$ of $V$ onto $S / \mathscr{Y}$; further if $S / \mathscr{Y}$ is finite, $V$ also can be chosen finite.

Let $T=\{(A, v) \in S / \mu \times V: A=s \mu, v \phi=s \mathscr{Y}$ for some $s \in S\}$. Then $T$ is easily seen to be a regular subsemigroup of $S / \mu \times V$. Let $\sigma$ denote the minimum group congruence on $V$ and define $\psi: T \rightarrow V / \sigma$ by $(A, v) \psi=v \sigma$. Then $\psi$ is a homomorphism of $T$ onto a group. Suppose $(A, v) \psi=1$ where 1 denoted the identity of $V / \sigma$; then since $V$ is $E$-unitary, $v^{2}=v$ so that $s \mathscr{Y}$ is idempotent. By (1), this implies $s^{2}=s$ so that $(A, v)$ is idempotent. Hence $T$ is E-unitary.

Now, for $(A, v) \in T, \operatorname{set}(A, v) \theta=s$ if $A=s \mu, v \phi=s \mathscr{Y}$. Then, since $\mathscr{Y} \cap \mu=\Delta, \theta$ is well defined and is a homomorphism of $T$ onto $S$. Suppose $(A, v) \theta=e=e^{2}$, then $A=e \mu$ and so, because $\mu$ is idempotent separating, $\theta$ is idempotent separating.

Lemma 2.2. Let $S$ be an E- unitary regular semigroup. Then $\mathscr{H} \cap \sigma=\Delta$ on $S$, where $\sigma$ is the minimum group congruence on $S$.

Proof. Let $(a, b) \in \mathscr{H} \cap \sigma$ and let $a^{\prime}, b^{\prime}$ be inverses for $a, b$ respectively such that $a a^{\prime}=b b^{\prime}, a^{\prime} a=b^{\prime} b$. Then $a b^{\prime} \mathscr{H} a a^{\prime}$ and $\left(a b^{\prime}, b b^{\prime}\right) \in \sigma$. Hence, since $S$ is $E$-unitary, $a b^{\prime}=a a^{\prime}$. Similarly $b^{\prime} a=b^{\prime} b$. Thus

$$
a=a a^{\prime} a=a b^{\prime} a=a b^{\prime} b=a a^{\prime} b=b b^{\prime} b=b .
$$

Theorem 2.3. Let $S$ be a finite orthodox semigroup. Then $S \in \mathscr{G}$ if and only if $\mathscr{H}$ is $a$ congruence on $S$.

Proof. Suppose that $\mathscr{H}$ is a congruence on $S$. By Lemma 2.1, there is a finite $E$-unitary regular semigroup $T$ and an idempotent separating homorphism $\theta$ of $T$ onto $S$. By Lemma 2.2, $T$ can be embedded in $T / \mu \times T / \sigma$ where $\mu$ is the maximum idempotent separating congruence on $T$ and $\sigma$ is the minimum group congruence. But, since $\theta$ is idempotent separating, $T / \mu \approx S / \mu=S / \mathscr{H}$ since $\mathscr{H}$ is a congruence. That is, $S$ divides $S / \mathscr{H} \times T / \sigma$ so that $S \in \mathscr{G}$.

The converse is immediate from Proposition 1.6.

Corollary 2.4. Let $S$ be a finite semigroup which is a union of groups. Then $S \in \mathscr{G}$ if and only if $S$ is an orthodox band of groups.

Proof. Suppose $S \in \mathscr{G}$ and let $e, f$ be idempotents. Then $e f$ belongs to a subgroup of $S$. Since $I G(S)$ is aperiodic, this implies ef is idempotent. Hence $S$ is orthodox and by Proposition 1.6, $\mathscr{H}$ is a congruence on $S$. That is, $S$ is an orthodox band of groups.

The converse is immediate from Theorem 2.3.

Corollary 2.5. The genus of finite semigroups generated by finite bands and finite groups is the genus of finite orthodox bands of groups. 
Proof. If $S$ divides the direct product of a finite band and a finite group, then $S$ is an orthodox band of groups. Conversely, if $S$ is a finite orthodox band of groups then, by the proof of Theorem $2.3, S$ divides $G \times S / \mu$ for some finite group $G$. But, since $\mathscr{H}$ is a congruence on $S, S / \mu$ is a band.

Remark 2.6. The strategy involved in the proof of Theorem 2.3 is the following. Given a finite regular semigroup $S$, find a finite regular semigroup $T$, on which $\mathscr{H} \cap \sigma=\Delta$ and an idempotent separating homomorphism $\theta$ of $T$ onto $S$.

Suppose now that such $T, \theta$ exists and let $e_{1}, \ldots, e_{r}$ be idempotents in $T$; let $w=e_{1} \ldots e_{r}$. Then, since $T$ is finite, $w^{n}, w^{n+1}$ belong to a subgroup of $T$ for some $n \geqq 1$. Further, since $w$ is a product of idempotents $\left(w^{n}, w^{n+1}\right) \in \sigma$. Thus, since $w^{n} \mathscr{C} w^{n+1}$, it follows from $\mathscr{H} \cap \sigma=\Delta$ that $w^{n}=w^{n+1}$. Hence $((1)$, Theorem III, 7.6) $I G(T)$ is aperiodic and consequently $I G(S)$ is aperiodic. We therefore pose the problem: if $S$ is a finite regular semigroup in which $I G(S)$ is aperiodic, does there exist a finite regular semigroup $T$, with $\sigma \cap \mathscr{H}=\Delta$ on $T$, and an idempotent separating homomorphism of $T$ onto $S$ ?

\section{REFERENCES} 1976).

(1) S. EILenberg, Automata, Languages and Machines, Vol B (Academic Press New York,

(2) T. E. Hall, Orthodox semigroups, Pacific J. Math. 39 (1971), 677-686.

(3) D. B. MCALISTER, Groups, semilattices and inverse semigroups, Trans. American Math. Soc. 192 (1974), 1-18.

(4) W. D. MuNN, Fundamental inverse semigroups, Q. J. Math. Oxford (2), 21 (1970), 157-170.

(5) K. S. S. NAMBOORIPAD, Structure of regular semigroups (Dissertation, University of Kerala, Karivattom, Trivandrum, India, 1973).

(6) J. RHODES, Some results on finite semigroups, J. Algebra 4 (1966), 471-504.

DEPARTMENT OF MATHEMATICAL SCIENCES

NORTHERN ILLINOIS UNIVERSITY

DEKALB, ILLINOIS 60115 